\title{
Coordinated Cross-Talk Between the Myc and Mlx Networks in
}

\section{Liver Regeneration and Neoplasia}

Key words: Adenoma, $\beta$-catenin, ChREBP, Hepatoblastoma, Hepatocellular Carcinoma, Hippo,

MondoA

Running Title: Cross-Talk Between Myc and Mlx Networks

Huabo Wang ${ }^{1}$, Jie Lu ${ }^{1}$, Frances Alencastro ${ }^{2,3}$, Alexander Roberts ${ }^{1}$, Julia Fiedor ${ }^{1}$, Patrick Carroll ${ }^{5}$, Robert N. Eisenman ${ }^{5}$, Sarangarajan Ranganathan ${ }^{6}$, Michael Torbenson ${ }^{7}$, Andrew W. Duncan ${ }^{2,3,4}$ and Edward V. Prochownik 1,4,8,9,10

1Division of Hematology/Oncology, UPMC Children's Hospital of Pittsburgh, Pittsburgh PA;

${ }^{2}$ Department of Pathology, UPMC; ${ }^{3}$ McGowan Institute for Regenerative Medicine, UPMC, Pittsburgh, PA; ${ }^{4}$ Pittsburgh Liver Research Center, University of Pittsburgh, Pittsburgh, PA; ${ }^{5}$ Basic Sciences Division, Fred Hutchinson Cancer Research Center, Seattle, WA 98109; ${ }^{6}$ Department of

Pathology, Cincinnati Children's Hospital, Cincinnati, OH, 45229; ${ }^{7}$ Department of Laboratory

Medicine and Pathology, The Mayo Clinic, Rochester, MN, 55905; ${ }^{8}$ The UPMC Hillman Comprehensive Cancer Center; ${ }^{9}$ The Dept. of Microbiology and Molecular Genetics, The University of Pittsburgh Medical Center, Pittsburgh PA 15224.

${ }^{10}$ Contact information:

Edward V. Prochownik

Children's Hospital of Pittsburgh

Division of Hematology/Oncology

Rangos Research Center, Room 5124

4401 Penn Ave.

Pittsburgh, PA 15224

Tel: 412-692-6795

Email: procev@chp.edu 


\section{Abstract}

The Myc bHLH-ZIP transcription factor is deregulated by most cancers. As a heterodimer with the bHLH-ZIP protein Max, Myc regulates target genes that contribute to metabolism and proliferation. This "Myc Network" cross-talks with the "Mlx Network" comprised of the Myc-like bHLH-ZIP proteins MondoA and ChREBP and the Max-like bHLH-ZIP protein Mlx. This "Extended Myc Network" regulates genes with both common and distinct functions. We have generated hepatocytes lacking Mlx $(m / x K O)$ or Mlx+Myc (double KO or DKO) and quantified their abilities to replace dying hepatocytes in a murine model of Type I tyosinemia. We find that this function deteriorates as the Extended Myc Network is progressively dismantled. Genes dysregulated in $m / x K O$ and DKO hepatocytes include those involved in translation and mitochondrial function. The Myc and Mlx Networks thus cross-talk with the latter playing a disproportionate role. mycKO and $m / x K O$ mice also develop non-alcoholic fatty liver disease and $m / x K O$ and DKO mice develop extensive hepatic adenomatosis not observed in wild-type, mycKO, chrebpKO or mycKOxchrebpKO mice. In addition to demonstrating cooperation between the Myc and Mlx Networks, this study reveals the latter to be more important in maintaining metabolic and translational homeostasis, while concurrently serving as a suppressor of benign tumorigenesis. 


\section{Introduction}

c-Myc (Myc) is a bHLH-Zip transcription factor that regulates numerous target genes, which collectively support survival, proliferation, metabolism, and translation (Meyer and Penn 2008; van Riggelen et al. 2010; Carroll et al. 2015; Stine et al. 2015; Wahlstrom and Henriksson 2015; Carroll and Diolaiti 2016; Carroll et al. 2018; Carroll et al. 2020) . Positive regulation involves Myc's direct sequence-specific DNA binding in heterodimeric association with its obligate bHLH-Zip partner, Max (van Riggelen et al. 2010; Wahlstrom and Henriksson 2015). This occurs at canonical E-box elements that typically reside in proximal promoters (Dang et al. 2006; Kuznetsov et al. 2010; Levens 2013; Sabo and Amati 2014). Bound Myc-Max heterodimers recruit an assortment of transcription co-factors and chromatin modifiers such as histone acetylases and methyltransferases that collectively increase chromatin accessibility, relieve transcriptional pausing and increase the rate and efficiency of mRNA elongation (Liu and Levens 2006; Diolaiti et al. 2015; Wilde and Ayer 2015; Kalkat et al. 2017; Tu et al. 2018). Down-regulation of these genes, often occurring during cellular quiescence or differentiation, involves a reduction in Myc levels and a shift to E-box occupancy by heterodimers now comprised of Max and members of the transcriptionally repressive bHLH-ZIP Mxd family that includes Mxd1-4 and the less related Mnt and Mga factors (McArthur et al. 1998; Grandori et al. 2000; Carroll et al. 2018). Together, their binding reverses the chromatin modifications mediated by Myc-Max binding and restore transcriptional repression. Negative regulation by Myc is more indirect and involves interaction with and inhibition of positively-acting transcription factors such as Miz1 and Sp1 (Gartel and Shchors 2003; Herkert and Eilers 2010). The loss of transcriptional balance maintained by these different competing interactions is a feature of transformed cells, which 
often deregulate and/or over-express Myc (Nesbit et al. 1999; Meyer and Penn 2008; Levens 2013; Kalkat et al. 2017).

The "Myc Network" cross-talks and shares considerable regulatory overlap with a structurally related but distinct group of bHLH-Zip transcription factors that comprise the so-called "Mlx Network" (Billin and Ayer 2006; Carroll et al. 2015; Carroll and Diolaiti 2016; Carroll et al. 2018; Wang et al. 2018; Carroll et al. 2020). Classically believed to control target gene sets smaller and more functionally restricted than that overseen by Myc, the Myc-like equivalents of the Mlx Network include the glucose-regulated transcription factors ChREBP and Mondo A. Upon binding glucose, these proteins translocate to the nucleus, heterodimerize with the Max-like protein Mlx and bind to their target genes at "carbohydrate response elements" (ChoREs) comprised of tandem E-boxes separated by 5-6 nucleotides (Jeong et al. 2011; Poungvarin et al. 2015). While some genes have been identified that are dually regulated by both Myc and Mlx, their numbers and the degree to which this is a result of binding to shared versus separate sites has not been clearly delineated (Collier et al. 2007; Zhang et al. 2010). Although the Mlx Network is less widely implicated in tumorigenesis than the Myc Network, recurrent $M L X$ gene deletions nevertheless occur in as many as $10-20 \%$ of several human cancers, with the exact fraction correlating with the size of the deletion (https://portal.gdc.cancer.gov/genes/ENSG00000108788) (Carroll et al. 2015; Diolaiti et al. 2015; Carroll and Diolaiti 2016; Carroll et al. 2018; Schaub et al. 2018).

We previously explored the roles for these two networks in normal hepatocyte proliferation utilizing mice lacking the enzyme fumarylacetoacetate hydrolase (FAH). These animals serve as a model for Type I hereditary tyrosinemia in which FAH's inactivation allows toxic tyrosine catabolites to accumulate, leading to hepatic necrosis and liver failure (Grompe et al. 1995; Russo et al. 2001; Chinsky et al. 2017). Treatment with the drug 2-[2-nitro-4-trifluoromethylbenzoyl]-1,3- 
cyclohexanedione (NTBC) blocks the enzyme 4-hydroxyphenylpyruvic dioxygenase, which catalyzes the second step in tyrosine catabolism, thus preventing the accumulation of these toxic intermediates and circumventing the lethal consequences of FAH deficiency. Immuno-compromised FRG$\operatorname{NOD}\left(\right.$ fah $\left.^{-/}\right)$mice serve as a robust and sensitive animal model in which to evaluate the regenerative potential of fah+/+ hepatocytes. These are delivered intrasplenically followed by the cyclic withdrawal and reinstatement of NTBC over several months. As recipient hepatocytes accumulate toxic tyrosine intermediates and die, they are replaced by the donor cells, which expand as much as $50-100$-fold before eventually comprising up to $70 \%$ of the hepatic mass and allowing the recipients to achieve NTBC-independence (Edmunds et al. 2016; Wang et al. 2018). The FAH model thus places greater proliferative demands on regenerating hepatocytes than occurs after twothirds partial hepatectomy $(\mathrm{PH})$ that represents the gold standard for liver regeneration (Michalopoulos 2010). It also permits the simultaneous delivery of two or more competing populations of hepatocytes to the same recipient and thus for a direct comparison of their relative proliferative rates.

Using this approach, we previously showed that wild-type (WT) and myc-/- (mycKO) hepatocytes possess indistinguishable regenerative potential (Edmunds et al. 2016). This is quite different from most other cases where Myc's loss in either non-transformed or transformed cells or tissues profoundly suppresses proliferation (Landay et al. 2000; Trumpp et al. 2001; Dubois et al. 2008; Wang et al. 2008; Graves et al. 2012; Edmunds et al. 2016; Wang et al. 2018). In contrast, the regeneration of chrebp-/- (chrebpKO) hepatocytes was significantly impaired (Wang et al. 2018) and mycKO+chrebpKO hepatocytes were even more defective. These findings indicated that normal hepatocyte regeneration was more dependent upon the Mlx Network than the Myc Network and that the two pathways cross-talk and rescue one another's defects. 
We have now further explored the relationship between the Myc and Mlx Networks by generating two additional mouse strains. In the first (hereafter " $m / x K O$ "), deletion of the Mlx gene functionally inactivates the entire Mlx Network, including any potential rescue by MondoA that previously might have existed in chrebpKO mice (Wang et al. 2018). The second mouse strain, with a "double knockout" (hereafter "DKO") of both Myc and MIx. As reported below, hepatocytes from both strains are profoundly compromised in repopulating the livers of fah-/- recipients. They also suppress genes that control mitochondrial structure and function, ribosomal biogenesis and more general aspects of mRNA processing and translation. Older mice of both groups also develop nonalcoholic fatty liver disease (NAFLD) akin to that previously described in mycKO, chrebpKO or mycKO+chrebpKO mice. Finally and unexpectedly, nearly half of older $m / x K O$ and DKO mice develop multi-focal hepatic adenomas occasionally associated with small areas of hepatocellular carcinoma (HCC). These results further support the idea that the Myc and Mlx Networks cross-talk and cooperatively regulate a range of pathways related to energy metabolism, lipid balance, translation and proliferation. Finally, they reveal a heretofore unsuspected role for the Mlx Network as a suppressor of benign hepatic adenomatosis (Donato et al. 2015).

\section{Results}

Repopulation by mIxKO and DKO hepatocytes is severely compromised. Donor strains employed for competitive hepatocyte repopulation studies carried homozygous "floxed" alleles of the $m / x$ and/or myc genes (Supplemental Fig. S1) and expressed an albumin promoter-driven Tamoxifen-inducible CreER(Qu et al. 2014; Wang et al. 2018). Five daily i.p. injections of Tamoxifen were sufficient to inactivate each allele at the time of hepatocyte transplant 3-4 months later (Supplemental Fig. S2). 
Using FRG-NOD mice as recipients, we previously showed that WT donor hepatocytes outcompeted an equivalent number of chrebpKO hepatocytes whereas WT and mycKO hepatocytes competed equally (Grompe et al. 1995; Edmunds et al. 2016; Wang et al. 2018). Hypothesizing that mIxKO hepatocytes were even more defective, we delivered a total of $3 \times 10^{5}$ donor hepatocytes intrasplenically into recipient mice at a $\sim 1: 6 \mathrm{WT}: m / x K O$ ratio (Fig. 1A, B). After 24-28 wk of NTBC cycling, a number of recipients had died and no survivors had achieved NTBC independence, possibly as a result of the under-representation of WT hepatocytes. Indeed, quantification of the total donor population in the surviving recipients indicated that it comprised only $2-46 \%$ of all hepatocytes, which is both lower and more variable than typically achieved when mice are receive larger numbers of replication-competent donor cells (Fig. 1C) (Edmunds et al. 2016; Wang et al. 2018). Despite this low level reconstitution, the surviving donor hepatocytes were nearly all WT (Fig. 1D).

Although mycKO alone does not confer a replicative disadvantage to hepatocytes, chrebpKO is exacerbated by concurrent myc inactivation (Wang et al. 2018). This suggests that the Myc and Mlx Networks compensate for one another under certain circumstances. Because mycKO x chrebpKO hepatocytes still express MondoA (Wang et al. 2018), we asked whether its redundant function might rescue more prominent phenotypes. We therefore compared the replicative potential of a mixed population of WT and DKO hepatocytes (1:10 ratio) in which the latter cells have functionally inactivated both ChREBP and MondoA as a consequence of $m / x$ deletion. This experiment achieved a somewhat greater rate of transplant success, with more animals surviving and with recipient livers eventually containing $>50 \%$ donor hepatocytes (Fig. 1E). However, as before, virtually all these were of WT origin despite their initial minority status (Fig. 1F).

To determine more directly which KO population was more replicatively challenged, an additional competitive transplant experiment was performed using a 1:1 input ratio of $m / x K O$ and 
DKO donor hepatocytes. Overall survival was again low and no animals achieved NTBCindependence. This was consistent with the finding that $<2 \%$ of hepatocytes isolated from recipients were of donor origin (Fig. 1G). However, m/xKO hepatocytes showed an overwhelming survival advantage (Fig. $1 \mathrm{H})$ and comprised nearly $95 \%$ of the recovered donor population. Together, these results argue that, in a highly demanding model of liver regeneration (Edmunds et al. 2016; Jackson et al. 2017; Wang et al. 2018; Wang et al. 2021), Mlx loss and the ensuing functional inactivation of ChREBP and MondoA strongly compromise donor hepatocyte proliferation and/or survival. The additional loss of Myc compounds the regenerative defect.

Overlapping transcriptional dysregulation in mIxKO and DKO livers primarily involves genes with roles in mitochondrial structure and function and translation. Prior to comparing the whole transcriptome profiles of WT, $m / x K O$ and DKO livers, we confirmed that the latter two showed the expected dysregulation of their target genes. For this, we performed gene set enrichment analysis (GSEA) (Subramanian et al. 2005) with three collections of direct Myc target genes from the Molecular Signatures Data Base C2 collection (MSigDB) (http://www.gseamsigdb.org/gsea/msigdb/collections.jsp) and a 134 member set of Mondo A, ChREBP and Mlx direct target genes from the Qiagen Ingenuity Pathway Analysis Data set ( Supplemental Table S1). In the first case, two of the three Myc target gene sets were also significantly enriched in $m / x K O$ liver RNA seq profiles, indicating that some Myc-regulated genes are responsive to Mlx Network inactivation (Fig. 2A) (Billin and Ayer 2006; Diolaiti et al. 2015; Wang et al. 2018). In contrast, the enrichment of these transcripts was broader and more pronounced in DKO livers. In the second case, MondoA/ChREBP/Mlx target genes were significantly enriched in both $m / x K O$ and DKO livers as expected (Fig. 2B). These results confirmed that inactivation of the Myc and/or Mlx Networks were associated with unique as well as shared responses of their respective target genes. 
Volcano plots were used to compare individual gene expression profiles in the above livers and previously described mycKO, chrebpKO and mycKO x chrebpKO livers (Edmunds et al. 2016; Wang et al. 2018). In the latter three cases, few differences were identified relative to normal livers from similarly aged animals (differential expression 1.5-fold and $q<0.05$ ) (Fig. 2C). In contrast, $m / x K O$ and DKO livers showed up to 60 -fold more differences, with $94 \%$ of the deregulated genes in $m / x K O$ livers also being deregulated in DKO livers (Fig. 2D). These findings indicate that the Myc and Mlx Networks co-regulate many common targets and compensate for one another's inactivation. Consistent with this, Ingenuity Pathway Analysis (IPA) of the differentially expressed transcripts in DKO livers (and by extension mlxKO livers) found that six of the top seven most affected pathways involving these transcripts $\left(P \leq 10^{-15}\right)$ were those with roles in mRNA processing and translation, energy metabolism and mitochondrial structure and function (Fig. 2E). The apparent exception, involving a pathway related to coronavirus infection, contained numerous transcripts encoding ribosomal proteins. Collectively, these findings agreed with previous reports in livers, liver cancers and other cell types (Grandori et al. 2000; Li et al. 2005; Billin and Ayer 2006; Carroll et al. 2015; Diolaiti et al. 2015; Hsieh et al. 2015; Wilde and Ayer 2015; Carroll and Diolaiti 2016; Edmunds et al. 2016; Dolezal et al. 2017; Havula and Hietakangas 2018; Wang et al. 2018; Wang et al. 2021). Gene expression profiles compiled from the pathways shown in Fig. $2 \mathrm{E}$ and the MSigDB pointed to the down-regulation of transcripts encoding proteins involved in translation and mitochondrial structure and function in mycKO and $m / x K O$ livers and an even greater degree of down-regulation in DKO livers (Fig. 2F\&G) (Wang et al. 2018)).

Myc promotes the Warburg effect by virtue of up-regulating glucose transporters and most of the genes encoding glycolytic enzymes (Osthus et al. 2000; Dang et al. 2006; Kim and Dang 2006; Graves et al. 2012; Karim et al. 2012; Miller et al. 2012; Ward and Thompson 2012; Hsieh et al. 
2015; Thorens 2015; Wang et al. 2018). The expression of their genes was mostly unaltered in mIxKO or DKO livers (Table 1). Notable exceptions included the genes encoding the major hepatocyte glucose transporter Glut2/Slc2a2 and the glycolytic enzymes phosphofructokinase (PFK) and liver type pyruvate kinase (PKLR), all three of which are rate-limiting for glucose uptake and oxidation. Glut2/Slc2a2 is also required for the proper regulation of glucose-sensitive genes and for glucose-stimulated insulin secretion (Thorens 2015; Chadt and Al-Hasani 2020). Interestingly, none of these genes was dysregulated in livers lacking Myc; rather, they became increasingly downregulated as the disabling of the Mlx Network progressed.

Loss of the Extended Myc Network members causes NAFLD. Consistent with findings that the Myc and Mlx Networks both regulate genes and pathways involved in carbohydrate and lipid metabolism (Billin and Ayer 2006; Soucek and Evan 2010; Muller et al. 2014; Carroll et al. 2015; Wahlstrom and Henriksson 2015; Carroll and Diolaiti 2016; Edmunds et al. 2016; Wang et al. 2018), young mycKO, chrebpKO and mycKO x chrebpKO mice develop NAFLD (Edmunds et al. 2016; Goetzman and Prochownik 2018; Wang et al. 2018). However, our previous investigations did not determine if this was progressive or if the dual compromise of the Myc and Mlx Networks increased its severity. We therefore examined the livers of older (14-16 month) mycKO, chrebpKO, mycKO x chrebpKO, mlxKO and DKO mice to evaluate the extent of NAFLD. Relative to WT livers, all KO livers showed more intense Oil Red $\mathrm{O}$ staining but did not significantly differ from one another (Fig. 3). They also contained more total triglyceride than did the livers from younger mycKO, chrebpKO and $m y c K O x$ chrebpKO mice (Edmunds et al. 2016; Wang et al. 2018). These findings suggest that NAFLD appears earlier in $m / x K O$ and DKO mice but that mycKO mice eventually achieve a similar degree of severity. 
mIxKO and DKO mice develop age-related hepatic adenomatosis and occasional HCC. Unexpectedly, $36 \%$ of $m x / \mathrm{KO}$ and DKO animals (15 of 42) of both genders developed multiple smallmedium-sized hepatic neoplasms, which were never observed in WT, mycKO, chrebpKO or mycKO $x$ chrebpKO mice (Fig. 4A\&B) (Edmunds et al. 2016; Wang et al. 2018). These were mostly welldifferentiated and/or myxoid-type adenomas with numerous balloon cells, nuclear enlargement and microvesicular steatosis. A minority also showed small foci of well-differentiated HCC, which is sometimes associated with hepatic adenomas in humans (Fig. 4C and Supplemental Fig. S3A-I) (Donato et al. 2015; Torbenson 2018). Regardless of histology, adenomas showed more staining for Ki-67 than did the adjacent non-neoplastic liver parenchyma (Fig. 4D and Supplemental Fig. S3 JO).

Adenomas did not express Mlx protein, indicating that they did not originate from residual hepatocytes that had escaped $m / x$ locus excision and maintained a growth advantage (Fig. 4E). Normal livers and DKO adenomas also did not express detectable Myc protein nor did most $m / x K O$ adenomas. This was consistent with their slow growth rates and a likely consequence of their Extended Myc Network defects (Fig. 2C-G) (Edmunds et al. 2016; Wang et al. 2018). An exception was seen in a single large adenoma containing elements of HCC from an m/xKO mouse with marked hepatomegaly (liver weight $6.7 \mathrm{~g}$ or approx. three times normal) (Fig. 4E).

We performed RNAseq on five adenomas from $m / x K O$ mice and compared their transcriptome profiles to those of WT livers and 45 primary murine hepatoblastomas (HBs) generated by over-expressing the Hippo pathway terminal effector YAPS127A together with nine different patient-derived oncogenic mutations of $\beta$-catenin (Zhang et al. 2019). The transcriptional profiles of the adenomas were distinct from those of both livers and all HBs (Fig. 4F\&G). Adenomas did not over-express wild-type $\beta$-catenin or YAP as is common for HBs (Sylvester and Colnot 2014) 
but did dysregulate 15 of 22 transcripts that are aberrantly expressed in all murine HBs regardless of etiology and that correlate with survival in human HB and other cancers (Fig. 4H) (Prochownik 2021; Wang et al. 2021). Our results indicate that dismantling the Mlx Network, either alone or concurrently with Myc leads to the eventual emergence of multiple adenoma-like hepatic neoplasms (adenomatosis), which, like their human counterparts, can further acquire malignant, HCC-like features (Torbenson 2018).

\section{Discussion}

Most prior investigations into Myc's role in hepatic regeneration relied upon $\mathrm{PH}$ and yielded conflicting results that likely reflected differences in how regeneration was quantified(Edmunds et al. 2016). The short time span over which this occurs, when it was assessed and the fact that separate groups of mice were used may have further contributed to disparate outcomes. Because post-PH hepatocytes require fewer than two divisions to replace the missing mass, the model also poses a comparatively modest regenerative challenge. Indeed, even this low number overestimates the actual contribution made by hepatocytes given that about half the response to $\mathrm{PH}$ involves hypertrophy of the remaining liver remnant plus replicative contributions by non-hepatocyte components such as endothelial, Kupfer and stellate cells (Michalopoulos 2010; Miyaoka et al. 2012; Itoh and Miyajima 2014). In contrast, the FAH mouse model is associated with a more sustained and robust 50-100-fold expansion of pure populations of transplanted hepatocytes. It also provides a well-defined point at which stable regeneration can be assessed and a means by which competing donor can be simultaneously distinguished and quantified (Edmunds et al. 2016; Havula and Hietakangas 2018; Wang et al. 2018). Using this approach, we previously showed that the normal long-term proliferation of hepatocytes requires ChREBP but not of Myc, although the loss of both factors was additive (Edmunds et al. 2016; Wang et al. 2018). Even more pronounced dependencies 
upon these two factors were seen during HB tumorigenesis (Jackson et al. 2017; Wang et al. 2018; Wang et al. 2021), with HB growth being markedly impaired in both mycKO and chrebpKO livers and even more so in mycKO x chrebpKO livers. These findings implied a means of communication between the Myc and Mlx Networks, with each being able to at least partially rescue defects in the other(Carroll et al. 2015). They also demonstrated that Myc dependency becomes progressively more important as proliferative demand increases (Fig. 5).

Despite our previous transplant studies having been performed with input ratios of WT and KO hepatocytes different from those reported here (Edmunds et al. 2016; Wang et al. 2018), their outcomes are consistent and allow us to extend our conclusions regarding the relative importance of the Myc and Mlx Networks in liver regeneration. For example, our studies comparing WT and chrebpKO hepatocytes employed an input inoculum comprised of $62 \%$ of the latter population that was reduced by more than half following competitive repopulation (Wang et al. 2018). Our current results in which $m / x K O$ hepatocytes comprised $\sim 84 \%$ of donor cells but only $\sim 4 \%$ of the final population (Fig. 2D) provided strong evidence that the concurrent functional inactivation of both ChREBP and MondoA confers an even more striking proliferative disadvantage. This could be a direct effect due to the concurrent loss of ChREBP and MondoA binding to their respective target genes either individually or collaboratively with Myc thereby eliminating any possibility of rescue of one factor by another (Fig. 2A) (Billin and Ayer 2006; Collier et al. 2007; Zhang et al. 2010; Jeong et al. 2011; Poungvarin et al. 2015). An indirect effect that allowed Mxd1, Mxd4 and Mnt to more effectively suppress Myc target genes by increasing their association with Max also remains possible and is not excluded by the former scenario. The relative importance of these two models could vary among different target genes and at different times during repopulation. 
We also showed that the Myc + ChREBP knockout suppressed regeneration more than did the knockout of either individual factor, thereby corroborating previous evidence for inter-Network cross-talk (Billin and Ayer 2006; Diolaiti et al. 2015; Carroll and Diolaiti 2016; Carroll et al. 2018; Wang et al. 2018). In that study, which utilized nearly equal contributions of WT and mycKO x chrebpKO donor hepatocytes, the latter was reduced to $7.5 \%$ following repopulation (Wang et al. 2018). Though markedly impaired, the residual proliferative activity of these cells could have reflected the redundant function of MondoA (Fig. 1A). This is supported by two separate aspects of the current study. The first was the $\sim 45$-fold repopulation advantage of WT hepatocytes over DKO hepatocytes, whereas the second was the $\sim 10$-fold repopulation advantage of $\mathrm{m} / x \mathrm{KO}$ hepatocytes over DKO hepatocytes (Fig. 1F\&H). Collectively our current results indicate that both the Myc and Mlx Networks play distinct as well as redundant roles in normal hepatocyte proliferation. However much of the proliferative drive needed to sustain hepatocyte expansion in FAH mice is subsumed by the Mlx Network regardless of the Myc Network's status (Edmunds et al. 2016; Wang et al. 2018). This is supported by the progressive deterioration of repopulation potential as the Extended Network is gradually dismantled (Fig. 1C-H) (Wang et al. 2018).

In relatively quiescent cells or organs such as the liver, Myc is usually expressed at low levels and regulates relatively few genes in contrast to Mlx (Fig. 2C) (Hofmann et al. 2015; Edmunds et al. 2016; Wang et al. 2018). Myc's contribution to genome-wide transcription may therefore be better appreciated in tumors where its over-expression can activate genes with low-affinity binding sites that are otherwise non-physiologic targets (Soucek and Evan 2010; Levens 2013; Sabo and Amati 2014; Wang et al. 2018). Another plausible explanation for the seemingly modest transcriptional consequences of Myc loss in some normal tissues is that at least some Myc target gene expression is maintained by the Mlx Network with redundant contributions being made by MondoA and ChREBP 
(Hofmann et al. 2015; Edmunds et al. 2016; Wang et al. 2018). This is best emphasized, both in livers and tumors, when the Myc and Mlx Networks are both inactivated (Fig. 2C) (Wang et al. 2018).

In tumors, the Myc Network can positively regulate most of the genes encoding glycolytic enzymes and strongly contributes to the Warburg effect (Dang et al. 2006; Kim and Dang 2006; Graves et al. 2012; Ward and Thompson 2012; Hsieh et al. 2015; Stine et al. 2015; Dolezal et al. 2017; Wang et al. 2019b). Non-transformed but rapidly growing fibroblasts also demonstrate Mycdependent changes in glycolytic function in vitro (Graves et al. 2012; Wang et al. 2019b). In contrast, our transcriptomic studies have not revealed wide-spread roles for the Myc and Mlx Networks in maintaining glycolysis in vivo, which may reflect Myc's low-level expression, the relative quiescence of the normal liver and its greater reliance on fatty acid oxidation as an energy source (Dolezal et al. 2017; Jackson et al. 2017; Wang et al. 2018; Zhang et al. 2019). Nonetheless, among the most down-regulated genes in $\mathrm{mlxKO}$ and/or DKO livers, were glut2/s/c2a2, pfkl and pklr whose encoded proteins (glucose transporter 2, liver-type phosphofructokinase and pyruvate kinase L/R, respectively) are rate-limiting for glucose uptake and glycolysis (Table 1). In rat insulinoma cells, the pklr proximal promoter binds both ChREBP and Myc, with the former interacting with a ChoRE element and the latter binding elsewhere (Collier et al. 2007; Zhang et al. 2010). These results suggest that, in normal liver, glucose uptake and oxidation rely more on the Mlx Network (Fig. 5). However, in response to oncogenic transformation or rapid normal cell proliferation, more extensive transcriptional regulation of glucose uptake and its oxidation in response to elevated Myc is achievable (Nesbit et al. 1999; Meyer and Penn 2008; Dolezal et al. 2017; Kalkat et al. 2017; Wang et al. 2018; Mathsyaraja et al. 2019). This could have the additional benefit of sustaining cell division when micro-environmental glucose and oxygen were limiting and nutrient-dependent functions of MondoA and ChREBP were attenuated (Prochownik and Wang 2021). 
In contrast to large changes in the expression of small numbers of individual genes in $m / x \mathrm{KO}$ and DKO livers (Fig. 2C) less dramatic but coordinated changes in functionally related gene sets are more common and reveal more about the pathways supervised by the Extended Myc Network (Fig. 2A\&B) (Edmunds et al. 2016; Wang et al. 2018). These sets have been previously implicated as being Myc- and/or ChREBP/MondoA-regulated and support protein translation and mitochondrial structure and function (Fig. 2E-G) (Grandori et al. 2000; Graves et al. 2012; Edmunds et al. 2016; Dolezal et al. 2017; Goetzman and Prochownik 2018; Wang et al. 2018; Zhang et al. 2019). As with the individual glycolytic genes, the collective expression of these sets is progressively compromised as the Extended Myc Network is inactivated. The dramatic up-regulation of these pathways that accompanies hepatic tumorigenesis is also increasingly attenuated in response to inactivation of Myc, ChREBP and the combination of the two (Wang et al. 2018).

Myc and/or ChREBP inhibition is widely associated with lipid accumulation, which arises due to an overreliance on fatty acid oxidation and increased lipid uptake that exceeds the amount necessary to meet energy demands (Fig. 3) (Zirath et al. 2013; Muller et al. 2014; Wang et al. 2015; Edmunds et al. 2016; Wang et al. 2018). Young mice with hepatocyte-specific loss of ChREBP or combined Myc + ChREBP loss also accumulate more neutral lipid than do those with isolated Myc knockout (Wang et al. 2018). Although we did not serially follow these animals, our findings suggest that, early in life, the partial or complete inactivation of the Mlx Network promotes a more rapid genesis of NAFLD than does inactivation of Myc alone. Over time, however, lipid accumulation equalizes with little differences among the various $\mathrm{KO}$ groups being discernible (Fig. 3).

An unanticipated finding was the development of hepatic adenomatosis in nearly $40 \%$ of $m I x K O$ and DKO mice (Fig. 4A\&B). This is likely an underestimate since animals older than 14-16 mos. were not investigated and microscopic adenomas may have been over-looked in some 
instances. That similar neoplasms did not appear in WT, mycKO, chrebpKO or mycKO+chrebpKO mice makes it likely that complete Mlx Network inactivation is a pre-requisite for their development. While these neoplasms bear the hallmarks of bona fide human adenomas (Donato et al. 2015; Torbenson 2018), several features suggest more aggressive and malignant predilections, despite the lack of Myc expression in most. These include their multi-focality, their occasional HCC-like features and their robust Ki-67 expression (Fig. 4C\&E). In contrast, human adenomas, while wellknown for their occasional conversion to HCC, are typically few in number and have low-level Ki-67 expression (Torbenson 2018). Molecular features suggestive of more aggressive behavior include the dysregulation of 15 transcripts that we have recently identified as predicting inferior outcomes in human HBs and over a dozen other human cancer types (Fig. 4H) (Wang et al. 2021).

Recurrent $M L X$ gene deletions are associated with at least eight human cancer types and provide further reason to implicate the Mlx Network in the pathogenesis of hepatic adenomatosis (https://portal.gdc.cancer.gov/genes/ENSG00000108788). Genetic suppressors of hepatic adenomas and other benign tumors such as meningiomas, neurofibromas and uterine fibroids are well-documented but are distinct from their more notorious counterparts such as TP53, RB, PTEN, $A P C$ and BRCA1/2 that are associated with malignant tumors (Bluteau et al. 2002; Navarro et al. 2012; Lee et al. 2019; Williams et al. 2020). However, Mlx Network's role may also be more indirect and nuanced. For example, the impressive NAFLD that accompanies $m / x$ inactivation (Fig. 3 ) is a known predisposing factor for the development of both adenomas and HCC (Brunt et al. 2015; Marengo et al. 2016; Torbenson 2018). On the other hand, the failure of mycKO, chrebpKO or mycKO $x$ chrebpKO mice to develop adenomas or HCCs, despite their equally pronounced NAFLD as well as the fact that high-fat diets can actually suppress tumor growth (Wang et al. 2019a), argues for a more direct role for the Mlx Network in adenoma suppression. It will be of interest to 
determine whether $\mathrm{mlxKO}$ mice are more susceptible to transformation by other oncogenic stimuli even though the emergence of the ensuing tumors may be delayed.

In summary, we have shown that the Mlx Network is engaged in substantial biological and molecular cross-talk with the Myc Network and contributes more to normal hepatocyte expansion when tested in a demanding model of long-term liver regeneration (Grompe et al. 1995; Zhang et al. 2010; Edmunds et al. 2016; Jackson et al. 2017; Wang et al. 2018). Both networks, but the former in particular, alter the expression of numerous genes responsible for broad aspects of translation and energy generation by both aerobic and anaerobic pathways (Edmunds et al. 2016; Wang et al. 2018). The presumptive energy deficit that ensues as a consequence of compromising either or both of the networks is likely rectified by an increased uptake and oxidation of fatty acids (Wang et al. 2018; Zhang et al. 2019). However, these two processes are unbalanced leading to long-term NAFLD. The hepatic adenomatosis and occasional HCC seen in response to Mlx Network compromise suggests that the tumor suppressor-like activity of the Mlx Network counters the more pro-oncogenic tendencies of deregulated Myc. Our findings emphasize the elaborate orchestration of the Extended Myc Network in balancing energy demands and metabolism with normal and neoplastic proliferation.

\section{Materials and Methods}

Animal studies. All breeding, care, husbandry and procedures were approved by The University of Pittsburgh Department of Laboratory and Animal Resources (DLAR) and the Institutional Animal Care and Use Committee (IACUC) with standard animal chow and water provided ad libitum. C57BL6 mice expressing GFP have been previously described and were used as a source of wild-type (WT) control hepatocytes due to the ease with which the GFP gene could be identified (Wang et al. 2018). C57BL6 c-mycLoxP/LoxP mice have been previously described ( 
Supplemental Fig. S1A) (Baena et al. 2005; Edmunds et al. 2016; Wang et al. 2018). The generation of mice bearing a $1717 \mathrm{bp}$ deletion spanning exons 3-6 of the $m / x$ locus (Supplemental Fig. S1B) has also been recently described (Carroll et al. 2020). Transgenic mice expressing a fusion protein comprised of the hormone-binding domain of the estrogen receptor and Cre recombinase (CreER) and under the control of the albumin promoter that allows CreER to be activated in hepatocytes following tamoxifen exposure were a kind gift of Dr. Frank Gonzalez (Laboratory of Metabolism, Center for Cancer Research National Cancer Institute). The latter mice were bred to homozygosity with $m / x K O$ mice or $m y c^{L O x P / L o x P} \times m^{L}{ }^{L O x P / L o x P}$ mice (Edmunds et al. 2016; Wang et al. 2018). At weaning, mice were subjected to five daily i.p. injections of tamoxifen $(75 \mathrm{mg} / \mathrm{Kg}$ each) in corn oil. Several weeks later, hepatocytes were harvested as previously described (Edmunds et al. 2016; Wang et al. 2018). An aliquot of these was used for DNA isolation and to quantify the extent of myc and/or $m / x$ knockout (Supplemental Fig. S1). The remainder of the mycKO, chrebpKO or mycKO $\mathrm{x}$ chrebpKO hepatocytes were then combined in various proportions with WT hepatocytes and a total of $3 \times 10^{5}$ cells were injected intrasplenically into fah-/- FRG-NOD mice (Yecuris, Inc., Tualatin, OR)(Edmunds et al. 2016; Wang et al. 2018). All animals were maintained on 8 mg/L NTBC (Ark Pharm, Libertyville, IL) in their drinking water. After four days, NTBC was discontinued until mice lost approx. $20 \%$ of their body weight. NTBC was then re-instated until mice regained their ageappropriate weight. NTBC cycling was continued either until mice had become NTBC-independent (at least 20 wk post-transplantation) or until wk 28 in those cases where NTBC-independence was not achieved. Hepatocyte DNAs were then isolated from recipients and the TaqMan based approaches shown in Supplemental Fig. S1 were again used to determine the donor:recipient ratio and the relative contribution of each donor population(Edmunds et al. 2016; Wang et al. 2018). 
For gene expression profiling, the above-described $m y c^{\text {LoxP/LoxP }}, m / x^{\text {LoxP/LoP }}$ and $m y c^{\text {LoxP/LoxP }} \times$ m/x ${ }^{\text {LoxP/LoP }}$ mice were bred to B6.129-Gt(ROSA)26Sortm1(cre/ERT2)Tyj/J mice, which expresses CreER under the control of the ubiquitously-expressed ROSA26 promoter (Ventura et al. 2007). Excisional inactivation of each locus was initiated at the time of weaning and confirmed as described above. Liver RNAs were then obtained from mice that were approximately the same age as those used for hepatocyte transplants ( 5 mos).

Triglyceride assays. Total lipid was extracted from $\sim 50 \mathrm{mg}$ of liver using the Folch method (Folch et al. 1957). Total triglyceride content was then determined as described previously using the Free Triglyceride Reagent (Sigma-Aldrich, Inc. St. Louis, MO) (Jouihan 2012; Wang et al. 2018).

Histology, immuno-histochemistry and immuno-blotting. Fresh tissues sections were immediately fixed in formalin, embedded in paraffin and stained with hematoxylin-eosin (H\&E) as previously described (Edmunds et al. 2016; Dolezal et al. 2017; Wang et al. 2018). Oil Red O (ORO) staining and immuno-histochemistry on snap-frozen sections were also performed as previously described (Edmunds et al. 2016; Wang et al. 2018). Tissue samples for immuno-blotting were disrupted in SDS-PAGE lysis buffer containing protease and phosphatase inhibitors but lacking $\beta$ mercaptoethanol or Bromophenol Blue as previously described (Dolezal et al. 2017; Jackson et al. 2017; Wang et al. 2018; Zhang et al. 2019). Protein quantification was performed using the BCA reagent according to the directions of the supplier (Sigma-Aldrich, St. Louis, MO). Following $\beta$ mercaptoethanol (1\%) and Bromophenol Blue (10\%) addition, samples were boiled for $5 \mathrm{~min}$, dispensed into small aliquots and stored at -80C until ready for use. SDS-PAGE and semi-dry transfer to PVDF membranes (Sigma-Aldrich) was performed as previously described (Dolezal et al. 2017; Jackson et al. 2017; Wang et al. 2018; Zhang et al. 2019). Antibodies used for immunoblotting included rabbit monoclonals directed against Mlx and Myc (\#85570 and \#13987, Cell 
Signaling Technologies) and a mouse monoclonal antibodies against GAPDH (\#G8795, SigmaAldrich). A mouse monoclonal anti-Ki-67 antibody used for immuno-histochemistry was also from Cell Signaling Technologies (\#12202). Horseradish peroxidase secondary antibodies were from Santa Cruz Biotechnology (Santa Cruz, CA). All antibodies were used at the dilutions recommended by the suppliers. Immunoblots were developed using an enhanced chemiluminescent assay kit as directed by the supplier (SuperSignal ${ }^{\mathrm{TM}}$ West Pico Plus, Thermo-Fisher, Inc., Waltham, MA).

RNA Experiments RNAseq. RNAs were purified from five replica tissues of each group of mice using Qiagen RNeasy Mini Kit (Qiagen) followed by DNase digestion (Edmunds et al. 2016; Wang et al. 2018). RNA concentration and integrity was confirmed on an Agilent 2100 Bioanalyzer (Agilent Technologies, Foster City, CA) and only those samples with RIN values of $>8.5$ were used for sequencing. All subsequent analyses were performed as previously described (Wang et al. 2018; Zhang et al. 2019). Sample preparation and sequencing was performed on a NovaSeq 600 instrument (Illumina, Inc., San Diego, CA) by Novagene, Inc. (Sacramento, CA) and raw data were deposited in the National Center for Biotechnology Information (NCBI) Gene Expression Omnibus data base (GEO) (Accession no GSE181371, GSE130178 and GSE114634). Differential gene expression was assessed by three different approaches, namely EdgeR, CLC Genomics Workbench version 21 (Qiagen) and DeSeq2, as previously described (Wang et al. 2018; Wang et al. 2021). When low-abundance reads $(\mathrm{cpm}<1)$ were encountered for both comparisons, they were eliminated. Reads from FASTQ files were mapped to the GRCm38.p6 mouse reference genome using STAR (https://github.com/alexdobin/STAR/releases) version 2.7.5. BAM-formatted output was analyzed and transcript abundance was determined by featureCounts (http://bioinf.wehi.edu.au/featureCounts/). Where necessary, Ingenuity profiling Analysis (IPA) (Qiagen) was used to classify transcripts into pathways whose significance was adjusted for false 
discovery using the Bonferonni-Hochberg correction. We further utilized Gene Set Enrichment Analysis (GSEA)(Subramanian et al. 2005) to identify alterations of functionally-related groups of transcripts from the Molecular Signatures Database (mSigDB) C2 collection (v.7.4) (http://www.gseamsigdb.org/gsea/msigdb/index.jsp) or from the MitoProteome Data base (http://www.mitoproteome.org). Volcano plots were generated using the R software package ggplot2 (https://ggplot2.tidyverse.org/) with significant differences between samples being defined as having fold differences $>1.5$ and false discovery rates $<0.05$. Heat maps were generated using the ComplexHeatmap package (version 2.6.2, https://bioconductor.org/packages/release/ bioc/html/ComplexHeatmap.html). Statistical Analysis were performed with R software v4.0.3 (R Foundation for Statistical Computing, Vienna, Austria) and GraphPad Prism v9.00 (GraphPad Software Inc., USA).

\section{Competing Interests Statement}

The authors declare no competing interests

\section{Acknowledgements}

This work was supported by NIH RO1 grants DK103645 to A.W.D., CA174713 to E.V.P. and NCI grant R35 CA231989 to R.N.E and P.C. E.V.P. was also supported by a Mellon Foundation Grant and a UPMC CHP Research Advisory Committee Seed Award. A.R. and J.F. were supported by The UPMC CHP Summer Research Undergraduate Fellowship Program. Analysis of RNAseq data was supported by The University of Pittsburgh Center for Research Computing.

\section{Author contributions}

Experimental design: E.V.P, H.W., A.D. 
Generation of mouse strains: P.C., R.N.E., H.W.

Hepatocyte transplantation: F.A., A.D.

Histopatholgy studies: M.T., S.R.

Biochemical studies: J.L., A.R., J.F.

RNAseq analyses: H.W.

Manuscript and figure preparation: E.V.P., H.W.

\section{References}

Baena E, Gandarillas A, Vallespinos M, Zanet J, Bachs O, Redondo C, Fabregat I, Martinez AC, de Alboran IM. 2005. c-Myc regulates cell size and ploidy but is not essential for postnatal proliferation in liver. Proc Natl Acad Sci U S A 102: 72867291.

Billin AN, Ayer DE. 2006. The Mlx network: evidence for a parallel Max-like transcriptional network that regulates energy metabolism. Curr Top Microbiol Immunol 302: 255-278.

Bluteau O, Jeannot E, Bioulac-Sage P, Marques JM, Blanc JF, Bui H, Beaudoin JC, Franco D, Balabaud C, Laurent-Puig P et al. 2002. Bi-allelic inactivation of TCF1 in hepatic adenomas. Nat Genet 32: 312-315.

Brunt EM, Wong VW, Nobili V, Day CP, Sookoian S, Maher JJ, Bugianesi E, Sirlin CB, Neuschwander-Tetri BA, Rinella ME. 2015. Nonalcoholic fatty liver disease. Nat Rev Dis Primers 1: 15080.

Camarda R, Williams J, Goga A. 2017. In vivo Reprogramming of Cancer Metabolism by MYC. Front Cell Dev Biol 5: 35. 
Carroll PA, Cheng PF, Freie BW, Kasinathan S, Gu H, Hedrich T, Dowdle JA, Venkataramani V, Ramani V, Raftery D et al. 2020. MLX balances metabolism and stress to suppress apoptosis and maintain spermatogenesis. bioRxiv: 2020.2012.2023.424063.

Carroll PA, Diolaiti D. 2016. A novel role for the extended MYC network in cancer cell survival. Mol Cell Oncol 3: e1026528.

Carroll PA, Diolaiti D, McFerrin L, Gu H, Djukovic D, Du J, Cheng PF, Anderson S, Ulrich M, Hurley JB et al. 2015. Deregulated Myc requires MondoA/Mlx for metabolic reprogramming and tumorigenesis. Cancer Cell 27: 271-285.

Carroll PA, Freie BW, Mathsyaraja H, Eisenman RN. 2018. The MYC transcription factor network: balancing metabolism, proliferation and oncogenesis. Front Med 12: $412-425$.

Chadt A, Al-Hasani H. 2020. Glucose transporters in adipose tissue, liver, and skeletal muscle in metabolic health and disease. Pflugers Arch 472: 1273-1298.

Chinsky JM, Singh R, Ficicioglu C, van Karnebeek CDM, Grompe M, Mitchell G, Waisbren SE, Gucsavas-Calikoglu M, Wasserstein MP, Coakley K et al. 2017. Diagnosis and treatment of tyrosinemia type I: a US and Canadian consensus group review and recommendations. Genet Med 19.

Collier JJ, Zhang P, Pedersen KB, Burke SJ, Haycock JW, Scott DK. 2007. c-Myc and ChREBP regulate glucose-mediated expression of the L-type pyruvate kinase gene in INS-1-derived 832/13 cells. Am J Physiol Endocrinol Metab 293: E48-56.

Dang CV, O'Donnell KA, Zeller KI, Nguyen T, Osthus RC, Li F. 2006. The c-Myc target gene network. Semin Cancer Biol 16: 253-264. 
Diolaiti D, McFerrin L, Carroll PA, Eisenman RN. 2015. Functional interactions among members of the MAX and MLX transcriptional network during oncogenesis. Biochim Biophys Acta 1849: 484-500.

Dolezal JM, Wang H, Kulkarni S, Jackson L, Lu J, Ranganathan S, Goetzman ES, Bharathi SS, Beezhold K, Byersdorfer CA et al. 2017. Sequential adaptive changes in a c-Myc-driven model of hepatocellular carcinoma. J Biol Chem 292: 10068-10086.

Donato M, Hamidian Jahromi A, Andrade AI, Kim R, Chaudhery SI, Sangster G. 2015. Hepatic adenomatosis: a rare but important liver disease with severe clinical implications. Int Surg 100: 903-907.

Dubois NC, Adolphe C, Ehninger A, Wang RA, Robertson EJ, Trumpp A. 2008. Placental rescue reveals a sole requirement for c-Myc in embryonic erythroblast survival and hematopoietic stem cell function. Development 135: 2455-2465.

Edmunds LR, Otero PA, Sharma L, D'Souza S, Dolezal JM, David S, Lu J, Lamm L, Basantani M, Zhang P et al. 2016. Abnormal lipid processing but normal longterm repopulation potential of myc-/- hepatocytes. Oncotarget 7: 30379-30395.

Folch J, Lees M, Sloane Stanley GH. 1957. A simple method for the isolation and purification of total lipides from animal tissues. J Biol Chem 226: 497-509.

Gartel AL, Shchors K. 2003. Mechanisms of c-myc-mediated transcriptional repression of growth arrest genes. Exp Cell Res 283: 17-21.

Goetzman ES, Prochownik EV. 2018. The Role for Myc in Coordinating Glycolysis, Oxidative Phosphorylation, Glutaminolysis, and Fatty Acid Metabolism in Normal and Neoplastic Tissues. Front Endocrinol (Lausanne) 9: 129. 
Grandori C, Cowley SM, James LP, Eisenman RN. 2000. The Myc/Max/Mad network and the transcriptional control of cell behavior. Annu Rev Cell Dev Biol 16: 653699.

Graves JA, Wang Y, Sims-Lucas S, Cherok E, Rothermund K, Branca MF, Elster J, Beer-Stolz D, Van Houten B, Vockley J et al. 2012. Mitochondrial structure, function and dynamics are temporally controlled by c-Myc. PLoS One 7: e37699.

Grompe M, Lindstedt S, al-Dhalimy M, Kennaway NG, Papaconstantinou J, TorresRamos CA, Ou CN, Finegold M. 1995. Pharmacological correction of neonatal lethal hepatic dysfunction in a murine model of hereditary tyrosinaemia type I. Nat Genet 10: 453-460.

Havula E, Hietakangas V. 2018. Sugar sensing by ChREBP/Mondo-Mlx-new insight into downstream regulatory networks and integration of nutrient-derived signals. Curr Opin Cell Biol 51: 89-96.

Herkert B, Eilers M. 2010. Transcriptional repression: the dark side of myc. Genes Cancer 1: 580-586.

Hofmann JW, Zhao X, De Cecco M, Peterson AL, Pagliaroli L, Manivannan J, Hubbard GB, Ikeno Y, Zhang Y, Feng B et al. 2015. Reduced expression of MYC increases longevity and enhances healthspan. Cell 160: 477-488.

Hsieh AL, Walton ZE, Altman BJ, Stine ZE, Dang CV. 2015. MYC and metabolism on the path to cancer. Semin Cell Dev Biol 43: 11-21.

Itoh T, Miyajima A. 2014. Liver regeneration by stem/progenitor cells. Hepatology 59: 1617-1626.

Jackson LE, Kulkarni S, Wang H, Lu J, Dolezal JM, Bharathi SS, Ranganathan S, Patel 
MS, Deshpande R, Alencastro F et al. 2017. Genetic Dissociation of Glycolysis and the TCA Cycle Affects Neither Normal nor Neoplastic Proliferation. Cancer Res 77: 5795-5807.

Jeong YS, Kim D, Lee YS, Kim HJ, Han JY, Im SS, Chong HK, Kwon JK, Cho YH, Kim WK et al. 2011. Integrated expression profiling and genome-wide analysis of ChREBP targets reveals the dual role for ChREBP in glucose-regulated gene expression. PLoS One 6: e22544.

Jouihan H. 2012. Measurement of Liver Triglyceride Content. Bio-protocol 2: e223.

Kalkat M, De Melo J, Hickman KA, Lourenco C, Redel C, Resetca D, Tamachi A, Tu WB, Penn LZ. 2017. MYC Deregulation in Primary Human Cancers. Genes (Basel) 8.

Karim S, Adams DH, Lalor PF. 2012. Hepatic expression and cellular distribution of the glucose transporter family. World J Gastroenterol 18: 6771-6781.

Kim JW, Dang CV. 2006. Cancer's molecular sweet tooth and the Warburg effect. Cancer Res 66: 8927-8930.

Kuznetsov VA, Singh O, Jenjaroenpun P. 2010. Statistics of protein-DNA binding and the total number of binding sites for a transcription factor in the mammalian genome. BMC Genomics 11 Suppl 1: S12.

Landay M, Oster SK, Khosravi F, Grove LE, Yin X, Sedivy J, Penn LZ, Prochownik EV. 2000. Promotion of growth and apoptosis in c-myc nullizygous fibroblasts by other members of the myc oncoprotein family. Cell Death Differ 7: 697-705.

Lee S, Karas PJ, Hadley CC, Bayley VJ, Khan AB, Jalali A, Sweeney AD, Klisch TJ, Patel AJ. 2019. The Role of Merlin/NF2 Loss in Meningioma Biology. Cancers 
(Basel) 11.

Levens D. 2013. Cellular MYCro economics: Balancing MYC function with MYC expression. Cold Spring Harb Perspect Med 3.

Li F, Wang Y, Zeller KI, Potter JJ, Wonsey DR, O'Donnell KA, Kim JW, Yustein JT, Lee LA, Dang CV. 2005. Myc stimulates nuclearly encoded mitochondrial genes and mitochondrial biogenesis. Mol Cell Biol 25: 6225-6234.

Liu J, Levens D. 2006. Making myc. Curr Top Microbiol Immunol 302: 1-32.

Marengo A, Rosso C, Bugianesi E. 2016. Liver Cancer: Connections with Obesity, Fatty Liver, and Cirrhosis. Annu Rev Med 67: 103-117.

Mathsyaraja H, Freie B, Cheng PF, Babaeva E, Catchpole JT, Janssens D, Henikoff S, Eisenman RN. 2019. Max deletion destabilizes MYC protein and abrogates Emicro-Myc lymphomagenesis. Genes Dev 33: 1252-1264.

McArthur GA, Laherty CD, Queva C, Hurlin PJ, Loo L, James L, Grandori C, Gallant P, Shiio Y, Hokanson WC et al. 1998. The Mad protein family links transcriptional repression to cell differentiation. Cold Spring Harb Symp Quant Biol 63: 423-433.

Meyer N, Penn LZ. 2008. Reflecting on 25 years with MYC. Nat Rev Cancer 8: 976-990. Michalopoulos GK. 2010. Liver regeneration after partial hepatectomy: critical analysis of mechanistic dilemmas. Am J Pathol 176: 2-13.

Miller DM, Thomas SD, Islam A, Muench D, Sedoris K. 2012. c-Myc and cancer metabolism. Clin Cancer Res 18: 5546-5553.

Miyaoka Y, Ebato K, Kato H, Arakawa S, Shimizu S, Miyajima A. 2012. Hypertrophy and unconventional cell division of hepatocytes underlie liver regeneration. Curr Biol 22: 1166-1175. 
Muller I, Larsson K, Frenzel A, Oliynyk G, Zirath H, Prochownik EV, Westwood NJ, Henriksson MA. 2014. Targeting of the MYCN protein with small molecule c-MYC inhibitors. PLoS One 9: e97285.

Navarro A, Yin P, Monsivais D, Lin SM, Du P, Wei JJ, Bulun SE. 2012. Genome-wide DNA methylation indicates silencing of tumor suppressor genes in uterine leiomyoma. PLoS One 7: e33284.

Nesbit CE, Tersak JM, Prochownik EV. 1999. MYC oncogenes and human neoplastic disease. Oncogene 18: 3004-3016.

Osthus RC, Shim H, Kim S, Li Q, Reddy R, Mukherjee M, Xu Y, Wonsey D, Lee LA, Dang CV. 2000. Deregulation of glucose transporter 1 and glycolytic gene expression by c-Myc. J Biol Chem 275: 21797-21800.

Poungvarin N, Chang B, Imamura M, Chen J, Moolsuwan K, Sae-Lee C, Li W, Chan L. 2015. Genome-Wide Analysis of ChREBP Binding Sites on Male Mouse Liver and White Adipose Chromatin. Endocrinology 156: 1982-1994.

Prochownik EV. 2021. Reconciling the Biological and Transcriptional Variability of Hepatoblastoma with Its Mutational Uniformity. Cancers (Basel) 13.

Prochownik EV, Wang H. 2021. The Metabolic Fates of Pyruvate in Normal and Neoplastic Cells. Cells 10.

Qu A, Jiang C, Cai Y, Kim JH, Tanaka N, Ward JM, Shah YM, Gonzalez FJ. 2014. Role of Myc in hepatocellular proliferation and hepatocarcinogenesis. J Hepatol 60: $331-338$.

Russo PA, Mitchell GA, Tanguay RM. 2001. Tyrosinemia: a review. Pediatr Dev Pathol 4: 212-221. 
Sabo A, Amati B. 2014. Genome recognition by MYC. Cold Spring Harb Perspect Med

4.

Schaub FX, Dhankani V, Berger AC, Trivedi M, Richardson AB, Shaw R, Zhao W, Zhang X, Ventura A, Liu Y et al. 2018. Pan-cancer Alterations of the MYC Oncogene and Its Proximal Network across the Cancer Genome Atlas. Cell Syst 6: $282-300$ e282.

Soucek L, Evan GI. 2010. The ups and downs of Myc biology. Curr Opin Genet Dev 20: 91-95.

Stine ZE, Walton ZE, Altman BJ, Hsieh AL, Dang CV. 2015. MYC, Metabolism, and Cancer. Cancer Discov 5: 1024-1039.

Subramanian A, Tamayo P, Mootha VK, Mukherjee S, Ebert BL, Gillette MA, Paulovich A, Pomeroy SL, Golub TR, Lander ES et al. 2005. Gene set enrichment analysis: a knowledge-based approach for interpreting genome-wide expression profiles. Proc Natl Acad Sci U S A 102: 15545-15550.

Sylvester KG, Colnot S. 2014. Hippo/YAP, beta-catenin, and the cancer cell: a "menage a trois" in hepatoblastoma. Gastroenterology 147: 562-565.

Thorens B. 2015. GLUT2, glucose sensing and glucose homeostasis. Diabetologia 58: 221-232.

Torbenson M. 2018. Hepatic Adenomas: Classification, Controversies, and Consensus. Surg Pathol Clin 11: 351-366.

Trumpp A, Refaeli Y, Oskarsson T, Gasser S, Murphy M, Martin GR, Bishop JM. 2001. c-Myc regulates mammalian body size by controlling cell number but not cell size. Nature 414: 768-773. 
Tu WB, Shiah YJ, Lourenco C, Mullen PJ, Dingar D, Redel C, Tamachi A, Ba-Alawi W, Aman A, Al-Awar R et al. 2018. MYC Interacts with the G9a Histone Methyltransferase to Drive Transcriptional Repression and Tumorigenesis. Cancer Cell 34: 579-595 e578.

van Riggelen J, Yetil A, Felsher DW. 2010. MYC as a regulator of ribosome biogenesis and protein synthesis. Nat Rev Cancer 10: 301-309.

Ventura A, Kirsch DG, McLaughlin ME, Tuveson DA, Grimm J, Lintault L, Newman J, Reczek EE, Weissleder R, Jacks T. 2007. Restoration of p53 function leads to tumour regression in vivo. Nature 445: 661-665.

Wahlstrom T, Henriksson MA. 2015. Impact of MYC in regulation of tumor cell metabolism. Biochim Biophys Acta 1849: 563-569.

Wang H, Dolezal JM, Kulkarni S, Lu J, Mandel J, Jackson LE, Alencastro F, Duncan AW, Prochownik EV. 2018. Myc and ChREBP transcription factors cooperatively regulate normal and neoplastic hepatocyte proliferation in mice. J Biol Chem 293: 14740-14757.

Wang H, Lu J, Dolezal J, Kulkarni S, Zhang W, Chen A, Gorka J, Mandel JA, Prochownik EV. 2019a. Inhibition of hepatocellular carcinoma by metabolic normalization. PLoS One 14: e0218186.

Wang H, Lu J, Kulkarni S, Zhang W, Gorka JE, Mandel JA, Goetzman ES, Prochownik EV. 2019b. Metabolic and oncogenic adaptations to pyruvate dehydrogenase inactivation in fibroblasts. J Biol Chem 294: 5466-5486.

Wang H, Lu J, Mandel JA, Zhang W, Schwalbe M, Gorka J, Liu Y, Marburger B, Wang J, Ranganathan S et al. 2021. Patient-Derived Mutant Forms of NFE2L2/NRF2 
Drive Aggressive Murine Hepatoblastomas. Cell Mol Gastroenterol Hepatol 12: 199-228.

Wang H, Mannava S, Grachtchouk V, Zhuang D, Soengas MS, Gudkov AV, Prochownik EV, Nikiforov MA. 2008. c-Myc depletion inhibits proliferation of human tumor cells at various stages of the cell cycle. Oncogene 27: 1905-1915.

Wang H, Sharma L, Lu J, Finch P, Fletcher S, Prochownik EV. 2015. Structurally diverse c-Myc inhibitors share a common mechanism of action involving ATP depletion. Oncotarget 6: 15857-15870.

Ward PS, Thompson CB. 2012. Metabolic reprogramming: a cancer hallmark even warburg did not anticipate. Cancer Cell 21: 297-308.

Wilde BR, Ayer DE. 2015. Interactions between Myc and MondoA transcription factors in metabolism and tumourigenesis. Br J Cancer 113: 1529-1533.

Williams EA, Wakimoto H, Shankar GM, Barker FG, 2nd, Brastianos PK, Santagata S, Sokol ES, Pavlick DC, Shah N, Reddy A et al. 2020. Frequent inactivating mutations of the PBAF complex gene PBRM1 in meningioma with papillary features. Acta Neuropathol 140: 89-93.

Zhang P, Metukuri MR, Bindom SM, Prochownik EV, O'Doherty RM, Scott DK. 2010. cMyc is required for the CHREBP-dependent activation of glucose-responsive genes. Mol Endocrinol 24: 1274-1286.

Zhang W, Meyfeldt J, Wang H, Kulkarni S, Lu J, Mandel JA, Marburger B, Liu Y, Gorka JE, Ranganathan S et al. 2019. beta-Catenin mutations as determinants of hepatoblastoma phenotypes in mice. J Biol Chem 294: 17524-17542.

Zirath H, Frenzel A, Oliynyk G, Segerstrom L, Westermark UK, Larsson K, Munksgaard 
Persson M, Hultenby K, Lehtio J, Einvik C et al. 2013. MYC inhibition induces metabolic changes leading to accumulation of lipid droplets in tumor cells. Proc Natl Acad Sci U S A 110: 10258-10263. 
Table 1. Relative expression levels of transcripts for rate-limiting steps in the glycolytic pathway.

\begin{tabular}{cccc} 
Liver Genotype & $\begin{array}{c}\text { Glucose transporter 2 } \\
\text { s/c2a2 }\end{array}$ & $\begin{array}{c}\text { Phosphofructokinase-liver type } \\
\text { pfkI }\end{array}$ & $\begin{array}{c}\text { Pyruvate kinase L/R } \\
\text { pklr }\end{array}$ \\
\hline WT & 1.00 & 1.00 & 1.00 \\
mycKO & NS* & NS & NS \\
chrebpKO & $0.30\left(q=5.0 \times 10^{-5}\right)$ & NS & $0.30\left(q=2.0 \times 10^{-7}\right)$ \\
mycKO x chrebpKO & $0.41(q=0.02)$ & NS & $0.29\left(q=7.0 \times 10^{-8}\right)$ \\
mIxKO & $0.10\left(q=8.3 \times 10^{-10}\right)$ & NS & $0.19\left(q=1.0 \times 10^{-10}\right)$ \\
DKO & $0.13\left(q=3.9 \times 10^{-9}\right)$ & $0.63(q=0.01)$ & $0.16\left(\mathrm{q}=2.4 \times 10^{-15}\right)$
\end{tabular}

*NS no significant difference $\mathrm{q}<0.05$ 


\section{Figures}

A

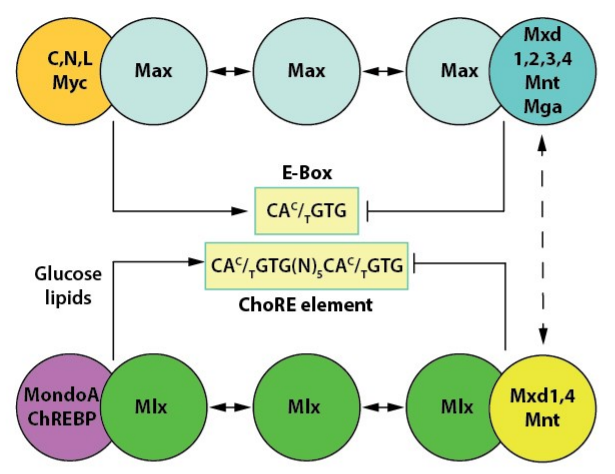

B

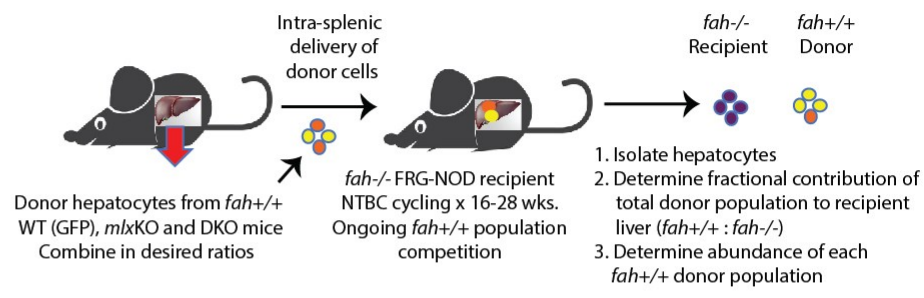

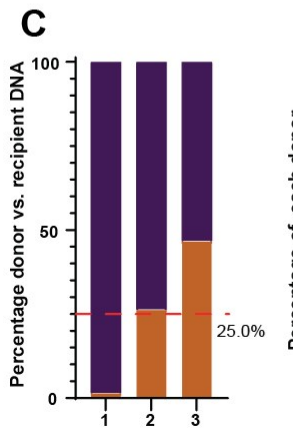

- Recipient

Donor

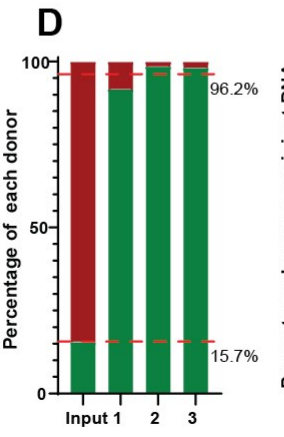

- WT

m m/xKO
E

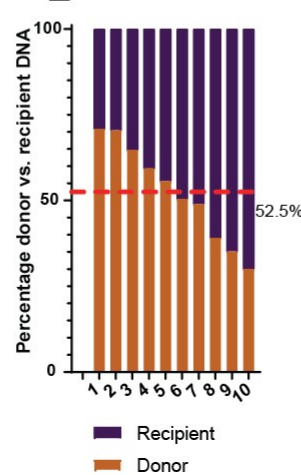

F

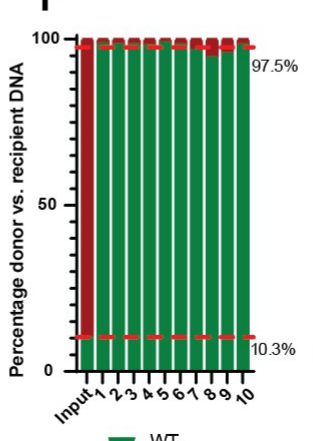

- WT
G

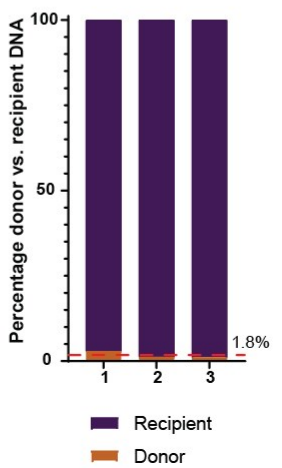

Wang_Fig1 
Fig. 1. WT hepatocytes outcompete $m / x K O$ and DKO hepatocytes in repopulation assays. (A). The Extended Myc Network. The top portion of the panel shows the Myc Network, comprised of Myc, Max, Mxd1-4, Mnt and Mga1 and their consensus E-box binding site. The bottom portion shows the Mlx Network and its E-box-related but more complex ChoRE binding site(Poungvarin et al. 2015; Jackson et al. 2017). Mlx interacts with the nutrient-regulated positive factors ChREBP and MondoA or the negative factors Mxd1, Mxd4 and Mnt (Billin and Ayer 2006; Diolaiti et al. 2015; Carroll and Diolaiti 2016). The latter cross-talk with the Myc Network (dotted arrow). (B). Hepatocyte transplantation strategy. Isolated fah+/+ WT or KO hepatocytes were mixed at the desired ratio and injected intrasplenically into FRG-NOD fah ${ }^{-/-}$mice maintained continuously on NTBC. NTBC cycling was continued until mice achieved NTBC independence or for 24-28 wks at which time total hepatocytes were isolated and the fractional representation of the total donor and recipient populations determined (Supplemental Fig. S1). The contribution of each donor set was then further determined and compared to that of the input inoculum. (C). Following intrasplenic injection of $3 \times 10^{5}$ donor hepatocytes comprised of a 1:6 ratio of WT and m/xKO cells, NTBC cycling was continued for 24-28 wks in the three animals that survived, with none achieving NTBC independence. Hepatocyte DNA was isolated from the transplanted animals and the percentage of recipient and total donor cells was determined. (D). DNAs from $\mathrm{C}$ were used to determine the ratio of the WT and $m / x K O$ donor populations. DNA from an aliquot of hepatocytes at the time of transplant was used to confirm the input donor cell ratio. (E). Transplants performed in FRG-NOD fah ${ }^{-/-}$mice using inocula containing a $\sim 1: 10$ ratio of WT:DKO cells. Hepatocytes isolated after $24-28$ wks of NTBC cycling showed that, on average, $52.5 \%$ of the total hepatocyte mass was comprised of the donor cells. $(F)$. Hepatocyte DNAs from E were used to determine the fraction of WT and $m / x K O$ donor hepatocytes. Lane 1 shows the $\sim 1: 10$ ratio of the initial input inoculum. (G). Transplants performed in FRG- 
NOD fah $^{-/-}$mice using inocula containing a $\sim 1: 1$ ratio of $m / x K O$ and DKO cells. Total hepatocytes isolated after 24-28 wks of NTBC cycling were evaluated for the fractional representation of total donor and recipient populations showing that, on average, $<2 \%$ of the total hepatocyte mass was of donor origin. $(H)$. Fractional make-up of the donor population from $G$. Lane 1 shows the $\sim 1: 1$ ratio of the input population. 
bioRxiv preprint doi: https://doi.org/10.1101/2021.08.05.455215; this version posted August 6, 2021. The copyright holder for this preprint (which was not certified by peer review) is the author/funder. All rights reserved. No reuse allowed without permission.

Wang_Fig2

A
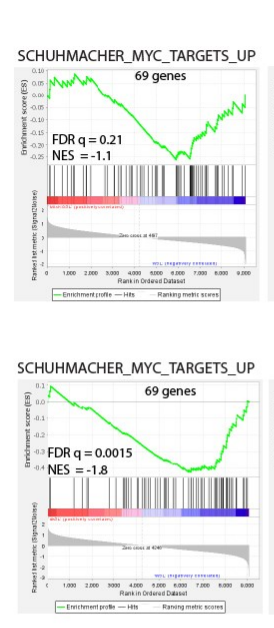

B

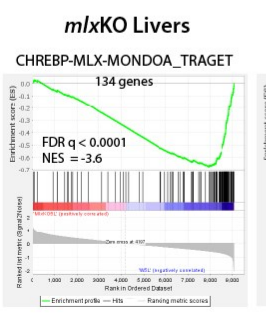

DKO Livers

CHREBP-MLX-MONDOA_TRAGET

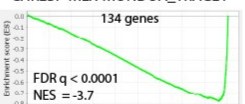

| |||||||||||||||| || |||||||||||

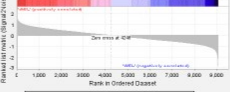

C
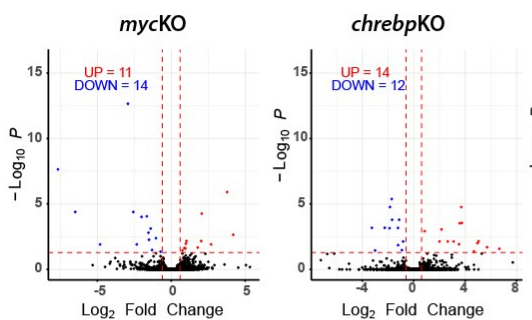

$\log _{2}$ Fold Change
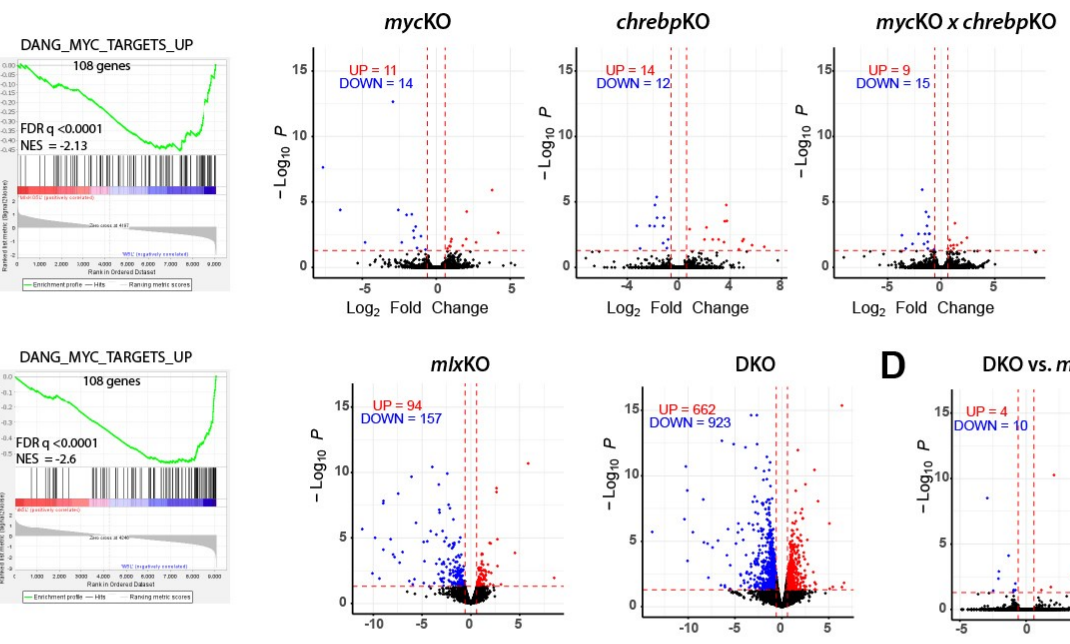

$\log _{2}$ Fold Change
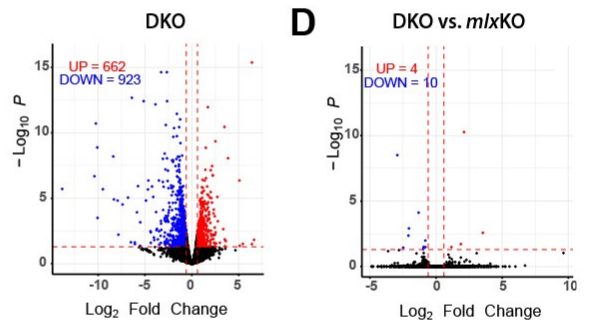

$\log _{2}$ Fold Change

\section{$\mathbf{F}$}
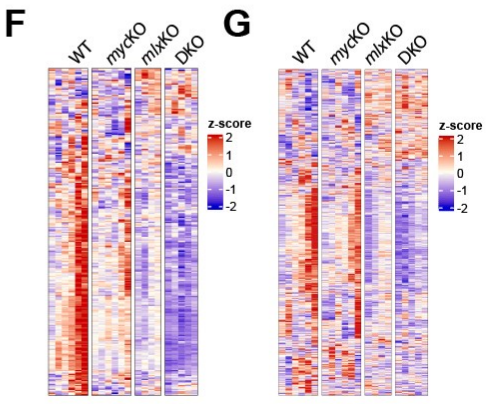
Fig. 2. Transcriptional dysregulation in response to Myc and/or Mlx Network inactivation. (A). GSEA performed on RNAseq data sets obtained from $m / x K O$ and DKO livers. Three sets of direct Myc target genes from the MSigDB Collection containing 69, 23 and 108 members, respectively, were used in the analysis, with expression levels being compared to those of WT livers ( $N=5$ samples per group). (B). A 134 member collection of direct MondoA, ChREBP and Mlx target genes from the Qiagen IPA data set was used in GSEA on the samples shown in A (Supplemental Table S1). (C). Volcano plots of differentially expressed genes in the indicated livers expressed relative to WT livers. Red and blue points = up-regulated and down-regulated, respectively relative to WT livers. chrebpKO and mycKO $\mathrm{x}$ chrebKO liver RNA seq results were obtained from Wang et al. (Wang et al. 2018). (D). Comparison of $m / x K O$ and DKO livers demonstrating the differential expression of only 14 transcripts. (E). IPA analysis showing the top seven dysregulated pathways in DKO livers. $(F)$. Heat maps of expression differences for 260 transcripts from the MSigDB C2 data base encoding ribosomal subunits and proteins involved in translation (https://www.gseamsigdb.org/gsea/msigdb/cards/REACTOME_TRANSLATION). (G). Expression differences for 605 transcripts encoding proteins comprising the mitochondrial proteome were compiled from the MitiProteome Data Base (http://www.mitoproteome.org). 
Wang_Fig 3
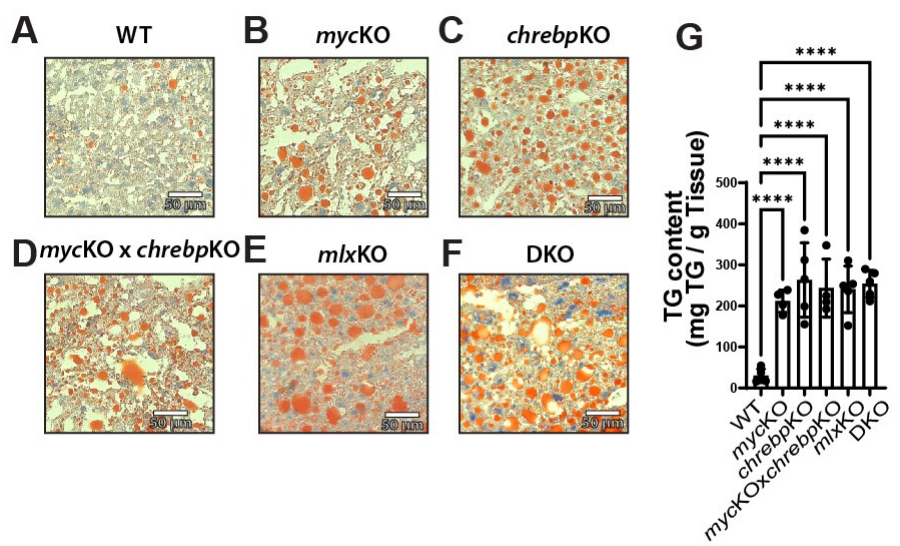

Fig. 3. Hepatic neutral lipid accumulation is a features of hepatocytes with compromised of the Extended Myc Network. (A-F). ORO stained sections of livers from 14-16 mo mice of the indicated genotypes. (G) Quantification of triglyceride levels in liver samples from A-F. 
A

\begin{tabular}{|c|c|}
\hline Genotype & $\begin{array}{c}\text { Mice with tumors } \\
\text { (adenomas)/total }\end{array}$ \\
\hline WT & $0 / 13$ \\
\hline mycKO & $0 / 4$ \\
\hline chrebpKO & $0 / 7$ \\
\hline mycKO x chrebpKO & $0 / 5$ \\
\hline mlxKO & $11 / 28$ \\
\hline DKO & $4 / 14$ \\
\hline
\end{tabular}

B

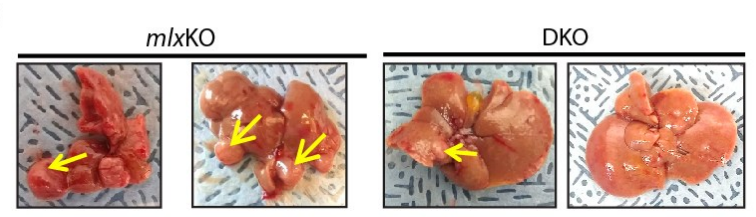

C

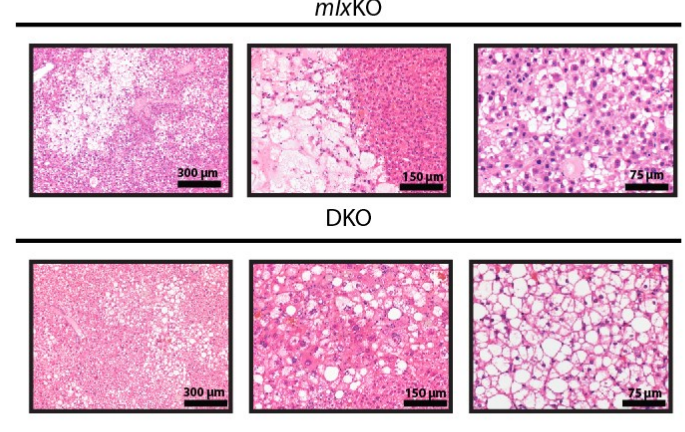

D H\&E

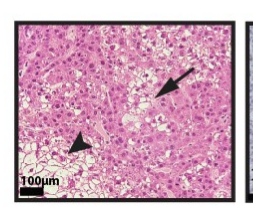

E

MIX

Myc

GAPDH

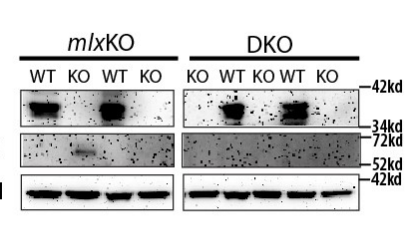

G
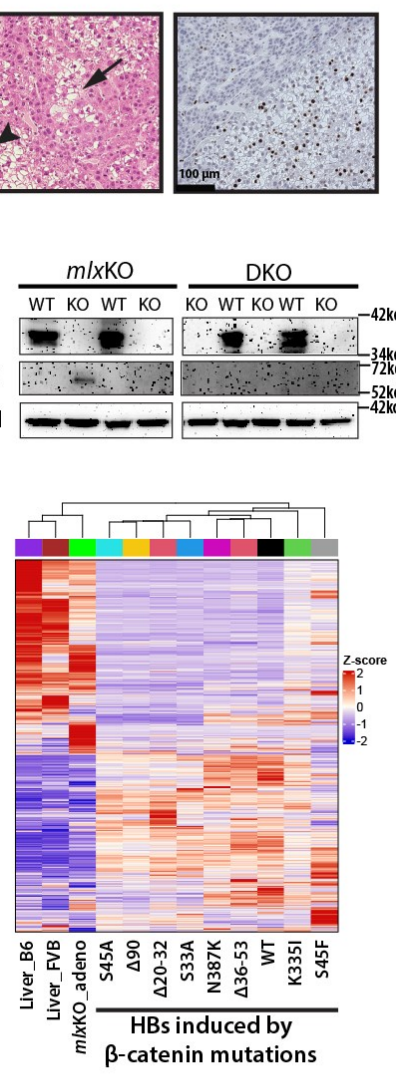

Wang_Fig4

F

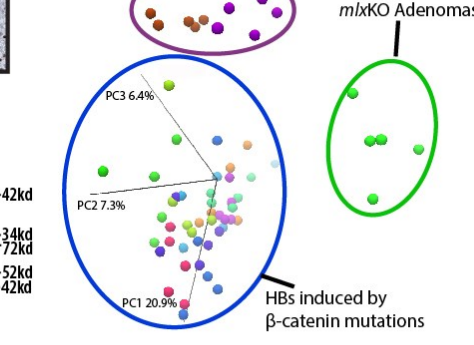

H

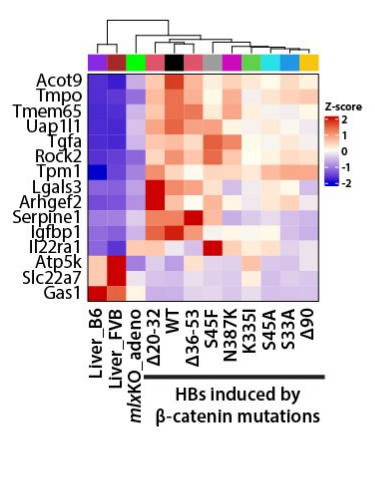


Fig. 4. Characterization of hepatic adenomas originating in $m / x K O$ and DKO livers. (A). Number of 14-16 mo mice of the indicated genotypes with visible liver tumors at the time of sacrifice. (B). Gross appearance of tumor-containing livers from A. Note that tumors were generally small but multifocal (arrows). (C). H\&E-stained sections showing the typical appearance of $m / x K O$ and DKO livers. The first two mlxKO images depict well-differentiated and myxoid-type adenomas, respectively, whereas the third panel depicts a focus of well-differentiated HCC embedded within an adenoma. The first two DKO images depict regions of microvesicular steatosis with balloon cells and nuclear enlargement, respectively whereas the third image shows another adenoma that is quite similar in appearance to those arising in m/xKO livers. See Supplemental Fig. S3A-I for additional H\&E-stained images. (D). H\&E and Ki-67 immuno-stained sections of the Myc-expressing adenoma from $D$ showing regions of inflammation (arrowhead), adjacent to those resembling welldifferentiated HCC (arrow). Ki-67 staining is more intense within the nodular adenoma (lower right) compared to adjacent normal liver (upper left). See Supplemental Fig. S3 J-O for additional examples of Ki-67-positive adenomas (E). Immuno-blots showing Mlx expression in WT livers and its absence in adenomas arising from $m / x K O$ and DKO livers. Only one large adenoma from an $m / x K O$ mouse with hepatomegaly expressed detectable levels of Myc (lane 2). (F). PCA of whole transcriptomes from WT livers, HBs generated by the over-expression mutant forms of $\beta$ catenin+YAPS127A and the above-described adenomas. (G). Whole transcriptome profiles of the tissues from F. Note that control liver samples are derived form two different strains, C57B6 and FVB. $(\mathrm{H})$. Heat maps of 15 of the 22 transcripts that are invariably dysregulated in murine HBs and correlate with poor outcomes in human HBs and other human cancers (Wang et al. 2021). 


\section{Wang_Fig5}

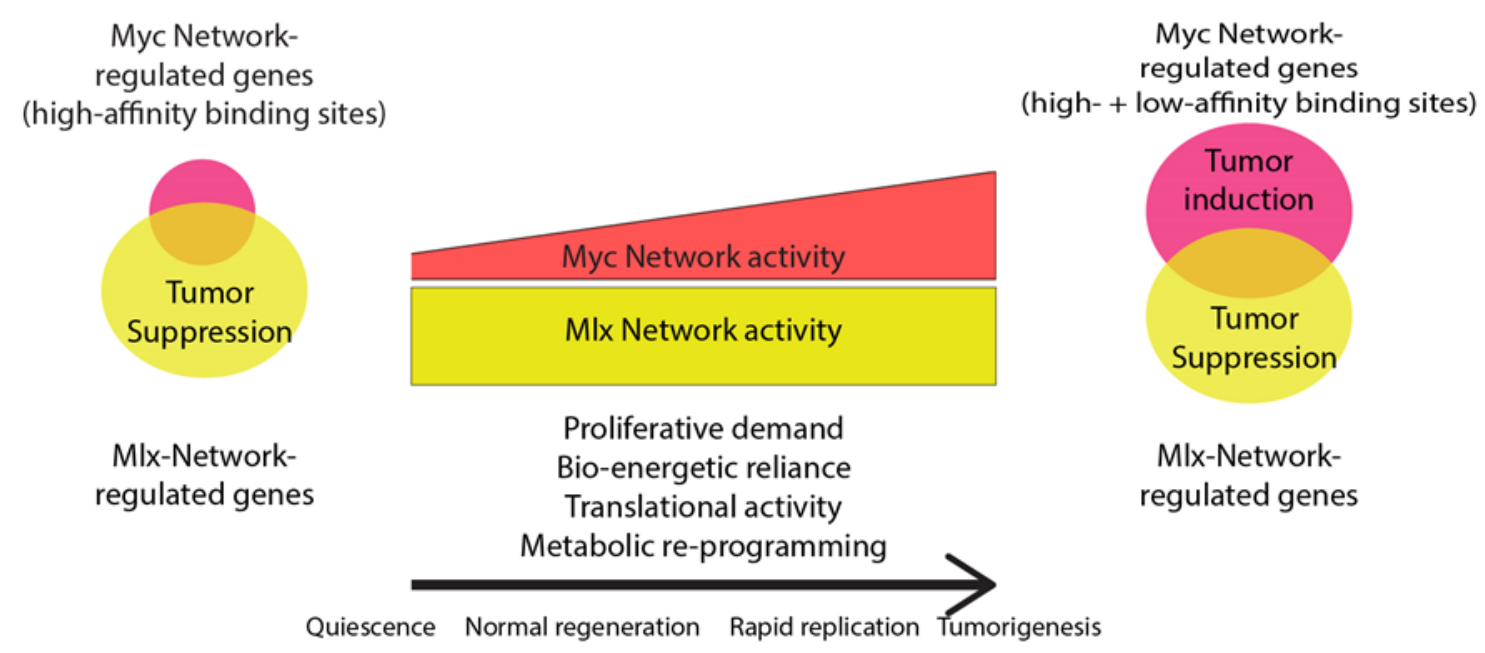

\section{Fig. 5. A model for gene regulation and control of normal and neoplastic proliferation by the}

Extended Myc Network. During quiescence, Myc levels are low and its target genes, which are relatively few in number (Fig. 2) and (Hofmann et al. 2015) tend to be those with high-affinity E-box binding sites. Slow and controlled replication, such as that occurring during the replacement of fah-/hepatocytes, is largely regulated via the Mlx Network (Edmunds et al. 2016; Wang et al. 2018). As replication increases or as cells become transformed, increased Myc expression (Dolezal et al. 2017; Wang et al. 2018) activates genes with low-affinity binding sites, including those encoding glycolytic enzymes that are collectively responsible for the Warburg effect as well as other metabolic pathways and functions that support increased energy demands (Billin and Ayer 2006; Graves et al. 2012; Hsieh et al. 2015; Carroll and Diolaiti 2016; Camarda et al. 2017; Carroll et al. 2018; Goetzman and Prochownik 2018) (Ward and Thompson 2012). Despite the rapid growth that can occur in response to the over-expression of Myc and mutant forms of $\beta$-catenin and YAP (Dolezal et al. 2017; Wang et al. 2018; Zhang et al. 2019) the Mlx Network, which supports this rapid growth, is proposed to also contribute to tumor suppression as well (Fig. 4). 